\title{
FORMULACIÓN DE UN MODELO DE PROGRAMACIÓN LINEAL ENTERA PARA LA ASIGNACIÓN DE AULAS DE CLASES EN UNA INSTITUCIÓN DE EDUCACIÓN SUPERIOR
}

\section{A LINEAR INTEGER PROGRAMMING MODEL FORMULATION FOR ASSIGNINING CLASSROOMS IN A HIGHER EDUCATION INSTITUTION}

\begin{abstract}
Ramiro Javier Saltos Atiencia, Ph.D. Doctor en Sistemas de Ingeniería - Gestión de Operaciones (Chile). Profesor de la Facultad de Ingeniería de la Universidad Espíritu Santo (Ecuador). https://orcid.org/0000-0001-6051-4028

rjsaltos@uees.edu.ec

Luis Enrique Benavides Castillo, M.Sc.

Magíster en Auditoría de Tecnologías de la Información (Ecuador). Profesor de la Facultad de Ingeniería de la Universidad Espíritu Santo (Ecuador). lebenavides@uees.edu.ec
\end{abstract}

\section{ARTÍCULO DE INVESTIGACIÓN}

Recibido: 24 de septiembre de 2019.

Aceptado: 30 de octubre de 2019.

\section{RESUMEN}

En este trabajo se propone un novedoso modelo de programación lineal entera para resolver el problema de asignación de aulas considerando como insumo fundamental la planificación académica de la Facultad de Ingeniería de una Institución de Educación Superior localizada en la provincia del Guayas, Ecuador. El modelo propuesto considera un conjunto de restricciones que deben ser satisfechas para que la asignación sea factible, que no existan cruces de materias para una misma aula en un bloque horario, no se exceda la capacidad del aula, entre otras. El modelo desarrollado fue implementado en el modelizador AIMMS Developer 4.68 y resuelto usando el solver IBM-CPLEX 12.9. La solución fue obtenida después de 3.61 segundos de ejecución satisfaciendo todas las 
restricciones y maximizando varios indicadores de calidad, tales como el nivel de utilización de las aulas, asignación de materias a aulas cuya infraestructura es afín con la naturaleza del contenido cubierto en la cátedra. Como conclusión se destaca la importancia de utilizar este tipo de métodos matemáticos para resolver problemas operativos propios de las Instituciones de Educación Superior dado que permiten obtener soluciones de alta calidad en mucho menos tiempo que su realización manual.

Palabras clave: Modelo de asignación de aulas, programación lineal entera, investigación de operaciones.

\section{ABSTRACT}

This paper proposes a new model of linear integer programming to solve the classroom assignment problem using as fundamental input the class-teacher scheduling plan of the Faculty of Engineering of a Higher Education Institution located in the province of Guayas, Ecuador. The proposed model has several constraints that must be satisfied so the assignment is feasible, such as: the classrooms are not assigned twice in the same time block, the capacity of the classrooms is not exceeded, among others, and was implemented in the AIMMS Developer 4.68 modeler and solved using the IBM-CPLEX 12.9 solver. The solution was obtained after 3.61 seconds of execution satisfying all the constraints and maximizing several quality indicators such as the level of use of the classrooms, allocation of class to classrooms whose infrastructure favors the nature of the content covered in the class, among others. As conclusion, the importance of using this type of mathematical methods to solve operational problems of higher education institutions is highlighted since they allow obtaining high quality solutions in much less time than their manual execution.

Keywords: Classrooms assignment model, linear integer programming, operations research.

\section{INTRODUCCIÓN}

En la actualidad, las instituciones de educación superior se enfrentan a diversas fuerzas que impactan su rendimiento académico, financiero y operacional. Entre estas fuerzas destacan los cambios en las leyes y reglamentos que rigen la educación superior en el 
Ecuador (CES, 2019; El Universo, 2019a), la demanda creciente de nuevos bachilleres que desean obtener un cupo para estudiar en alguna de las universidades del país, los retos impuestos por la era digital como la educación $100 \%$ en línea (EI Universo, 2019b), los nuevos modelos de acreditación impuestos por el órgano regulador (El Universo, 2019c), entre otras. Como cualquier otra organización, las universidades, en especial las universidades privadas o particulares, deben ser cada vez más competitivas en el exigente mercado de la educación superior, y para esto deben reducir sus costos y tener excelencia operacional.

En este contexto, uno de los principales problemas operacionales que semestre a semestre deben resolver las instituciones de educación superior es la planificación académica del periodo de clases que está por iniciar (Hernández, Miranda, y Rey, 2008; June, Obit, Leau, y Bolongkikit, 2019). La administración universitaria necesita planificar continuamente sus recursos de manera eficiente, tales como los maestros, las aulas. Esta decisión de gestión consiste en determinar, con base en la estimación de la demanda, qué cátedras, cuántos paralelos de cada cátedra y qué horarios deben ofertarse en el próximo periodo y tendrán un alto impacto en el costo de administrar una universidad y también en qué tan buenos serán los horarios resultantes (Lindahl, Mason, Stidsen, y Sørensen, 2018). Una vez definidos estos parámetros, el siguiente paso es asignar a cada cátedra y paralelo un profesor que la dicte, considerando tanto la competencia del profesor para dictar la asignatura, así como su disponibilidad horaria (Babaei, Karimpour, y Hadidi, 2015; Lewis, 2008). Tras realizar la asignación de las cátedras y paralelos a los respectivos profesores, se debe establecer en qué aulas se realizarán estas. Normalmente, las tareas anteriormente mencionadas suelen ejecutarse de manera manual sin la utilización de un sistema de apoyo a la toma de decisiones (DSS por sus siglas en inglés) que permita manejar de manera simultánea todas las posibles combinaciones que pueden existir para resolver el problema descrito (Weitz y Jelassi, 1992), por lo que el equipo responsable de este proceso suele requerir de uno o más meses de trabajo, en función del tamaño de la institución, hasta tener una planificación académica que satisfaga todas las restricciones impuestas por la casa de estudios.

A pesar de que del equipo de planificación académica de la casa de estudios superiores disponga de suficiente tiempo e información para la ejecución de esta tarea, dado que es 
un proceso manual no está exento de errores y su calidad puede ser inferior a una generada con el apoyo de un modelo de toma de decisiones cuantitativo y automatizado, a la vez que este puede ayudar a mejorar diferentes métricas de calidad al momento de construir la planificación académica (Hernández, Miranda, y Rey, 2008). Por esta razón es necesario que las instituciones educativas cuenten con sistemas de información de apoyo a la toma de decisiones que sea capaces de resolver problemas operacionales complejos tales como el descrito anteriormente de manera rápida y eficiente, minimizando la utilización del recurso humano de la institución, el cual debe estar destinado a otras actividades prioritarias tales como la preparación de material docente, instrumentos de evaluación, desarrollo y ejecución de proyectos de investigación, etc.

El problema de los horarios es organizar un número determinado de eventos en una cantidad de recursos (intervalos de tiempo, salas, etc.) y garantizar que se cumpla un conjunto predefinido de restricciones (Song, Liu, Tang, Peng, \& Chen, 2018), en este sentido, en el presente trabajo de investigación se propone un modelo matemático basado en programación lineal entera para resolver la tercera etapa de la planificación académica de una institución universitaria, esta es, la asignación de aulas utilizando como insumo la asignación de materias, paralelos y profesores ya realizada en las etapas anteriores. El modelo propuesto considera varios factores relevantes del problema tales como: el tipo de aula, su capacidad e infraestructura, naturaleza de las cátedras, cantidad de alumnos matriculados, preferencias de los profesores, entre otras. Como parte de los resultados, el modelo permite encontrar soluciones que mejoran el nivel de utilización de las aulas, disminuye la necesidad de prestar aulas a otras unidades académicas, las cátedras se dictan en aulas cuya infraestructura favorece la ejecución de esta, así como otros beneficios que se indican en la Sección 2.

El resto del documento está estructurado de la siguiente manera: En la Sección 2 se presenta la descripción del problema, la Sección 3 se establece los fundamentos teóricos y el estado del arte referente con problemas de calendarización en instituciones educativas, la Sección 4 desarrolla el modelo matemático y explica los resultados obtenidos, mientras que la Sección 5 concluye este artículo e indica las principales líneas de trabajo futuro a considerar. 
El problema que se aborda en el presente estudio corresponde al de asignar las aulas de clases el cual se considera un difícil problema de optimización combinatoria (Al-Betar, Khader, \& Zaman, 2012; Lewis, 2008), en este caso, en la Escuela de Computación y Telecomunicaciones de la Facultad de Ingeniería de una prestigiosa institución de educación superior localizada en la provincia del Guayas, Ecuador. Actualmente, la Escuela de Computación y Telecomunicaciones oferta dos carreras de pregrado y es responsable de las asignaturas pertenecientes al campo de la ciencias físicas y matemáticas, las cuales son transversales para varios de los programas de pregrado ofertados por la universidad. Como se mencionó en la Introducción, en este trabajo se considera únicamente la etapa de asignación de aulas, por lo cual, se asume que la asignación de docentes a las cátedras y a los bloques horarios se encuentra finalizada y disponible para su ejecución.

Usualmente, la complejidad de un problema varía ya que cada institución tiene sus propios requisitos (Goh, Kendall, \& Sabar, 2019), en este sentido, la institución elabora la planificación académica siguiendo los siguientes pasos, a saber:

1. El equipo responsable de la planificación realiza una estimación de la demanda potencial de alumnos que puede existir para cada una de las asignaturas que se ofertarán en el siguiente periodo académico. Con base en esta estimación, se decide cuáles asignaturas y cuántos paralelos de cada una ofertar en el nuevo periodo. La cantidad de paralelos se estima fácilmente debido a que en ninguna de las aulas pueden existir más de 33 estudiantes.

2. Una vez determinadas las asignaturas y la cantidad de paralelos por ofertar, se crean parejas de cátedra - paralelo que son ubicadas en los diferentes bloques horarios respetando ciertas restricciones importantes, tales como: (1) las asignaturas pertenecientes a los 5 primeros semestres de la malla curricular de las carreras deben dictarse en la jornada matutina. (2) las asignaturas pertenecientes al sexto semestre de la malla o superior deben asignarse en la jornada nocturna. (3) las asignaturas que pertenecen a un mismo nivel de la malla curricular, por cada paralelo, no deben cruzarse entre sí, es decir, los paralelos 01 de las materias del mismo nivel no debe tener un cruce de horarios, sin embargo, un paralelo 02 puede cruzarse con un paralelo 01 aunque sean cátedras de mismo semestre. 
3. Con la asignación de las cátedras y paralelos en los diferentes bloques horarios, el equipo solicita al cuerpo docente su disponibilidad horaria semanal. La mayoría del cuerpo docente de la universidad tiene una relación laboral de servicios profesionales prestados (profesores de tiempo parcial), es decir, estos profesores asisten al campus únicamente a dictar su clase y luego se retiran.

4. Considerando la disponibilidad horaria de los profesores de tiempo parcial, en conjunto con las preferencias de los profesores de tiempo completo, se asigna a cada pareja cátedra - paralelo un profesor ya sea de tiempo parcial o tiempo completo. Si un profesor de tiempo parcial se le asigna una pareja cátedra paralelo, entonces por disposiciones del vicerrectorado académico, este profesor debe ser asignado a por lo menos dos parejas cátedra - paralelo con un máximo de tres.

5. Una vez finalizada la asignación de los profesores a las distintas parejas cátedra paralelo, esta es ingresada al sistema de información de la universidad para que los alumnos puedan registrarse para el siguiente periodo académico. Aquí, la persona responsable de ingresar la planificación al sistema asigna de manera aleatoria las aulas a las cátedras tratando de respetar que no exista una doble asignación para las aulas.

Uno de los principales problemas que existen con el paso (5) radica en que la persona que asigna las aulas solo trata de que no exista cruce de horas para estas. Al realizar la asignación de manera manual, no considera factores relevantes que influyen en el desempeño de cada cátedra, tales como: (1) que el tamaño de la pizarra en el aula sea consecuente con la complejidad de los problemas abordados en la cátedra a la cual se le asigna dicha aula, (2) que la distribución de las sillas y mesas en el aula contribuya a una mejor visualización de la pizarra y del profesor, de tal manera que reduzca la desconcentración de los estudiantes y (3) algunos profesores influyen en el juicio del planificador de aulas, forzándolo a que le asignen las mejores aulas perjudicando la infraestructura donde operarán las demás. Normalmente, estos problemas no se pueden evitar por lo que se termina reasignando las aulas antes del inicio de clases, lo que ocasiona que los estudiantes tengan problemas, el primer día, para ubicar su clase. 
Como solución para reducir o eliminar las problemáticas anteriormente mencionadas, se hace necesario que la persona responsable de la asignación de las aulas cuente con una herramienta matemática y computacional que permita una rápida asignación de las aulas de tal forma que esta sea lo más justa y equitativa posible, dando prioridad al bienestar de los estudiantes que cursan las asignaturas.

\section{REVISIÓN TEÓRICA}

En la presente sección, se presentan los fundamentos teóricos que sirven como base para la modelación matemática del problema estudiado en esta investigación.

\section{Programación Lineal Entera}

La Investigación de Operaciones es una rama de las Matemáticas Aplicadas que estudia la formulación y resolución de problemas de optimización que representan situaciones del mundo real (Hillier, Lieberman, \& Osuna, 2010). Estos problemas suelen tener la siguiente estructura:

$$
\begin{aligned}
& \min z=f(x) \\
& \text { s.t: } \\
& g(x) \leq 0 \\
& x \geq 0
\end{aligned}
$$

En el modelo anterior, $x$ contiene las variables de decisión del problema, es decir, todos aquellos factores que son controlables por el tomador de decisiones, $f(x)$ representa la función objetivo que se desea optimizar, por ejemplo, funciones de utilidad o de costo, $g(x) \leq 0$ contiene el conjunto de restricciones que deben ser satisfechas por la solución del problema, tal como que no se supere la capacidad de un aula. Finalmente, $x \geq 0$ presenta la naturaleza que, por sentido común, deben tener las variables de decisión, como que no pueden existir tiempos negativos, entre otras.

Cuando la función objetivo y las restricciones de un problema de optimización satisfacen los criterios de linealidad, es decir, cumplen que:

1. $\forall x, y \in X f(x+y)=f(x)+f(y)$ 
2. $\forall c \in R, x \in X f(c x)=c f(x)$

y las variables de decisión toman valores reales, se tiene un problema de programación lineal (LP por sus siglas en inglés). Estos problemas suelen tener la siguiente estructura genérica:

$$
\begin{array}{ll}
\min z=\sum_{j=1}^{n} c_{j} x_{j} & \\
\sum_{j}^{\text {s.t }:} a_{i, j} x_{j} \leq b_{i} & \forall i=1,2 \ldots, m \\
x_{j} \geq 0 & \forall j=1,2, \ldots, n
\end{array}
$$

Donde $c_{j}, b_{i}, a_{i j} \in R$ para todo $i=1,2 \ldots, m$ y $j=1,2, \ldots, n$. Existen varios métodos matemáticos para resolver este tipo de problemas de optimización, el más famoso de ellos es el Método Simplex (Dantzig \& Thapa, 2006).

Cuando una o varias de las variables de decisión presentes en el problema de programación lineal deben tomar exclusivamente valores binarios ( 0 ó 1) o valores enteros, entonces se dicen que el problema de optimización es un problema de programación lineal entera (MIP por sus siglas en inglés). Una de las principales diferencias entre los problemas LP y los problemas MIP es que el espacio de soluciones factibles de este último puede crecer exponencialmente por todas las posibles combinaciones de soluciones que se pueden generar para el problema, aumentando considerablemente la dificultad para resolverlos, a pesar de la existencia de grandes métodos tales como los algoritmos de Branch\&Bound, Branch\&Price y Planos Cortantes (Wolsey, 1998). En el presente trabajo de investigación, el modelo que se formula y resuelve pertenece a esta familia de problemas de optimización.

\section{Problemas de calendarización}

Los problemas de optimización que se presentan con mayor frecuencia en el mundo real y que son muy relevantes a nivel operativo en cualquier organización son los problemas de calendarización. Un problema de calendarización consiste en la asignación de un conjunto de objetos, eventos o entidades a un conjunto de recursos escasos, tales como el tiempo y el espacio (Pinedo, 2016). Para el caso de las instituciones educativas, los objetos 
corresponden al conjunto de profesores, estudiantes, cátedras, entre otros, mientras que los recursos escasos suelen ser los bloques horarios y las aulas de clase (Nategh, Hosseinabadi, \& Balas, 2018). Cuando el problema de calendarización se origina de un contexto educativo, tales como colegios y universidades, el problema se denomina "Educational Timetabling Problem" (Hernández, Miranda, \& Rey, 2008; Babaei, Karimpour, \& Hadidi, 2015). Conforme al estudio realizado por Schaerf (1999) y, posteriormente, (Hosny, 2018), esta clase de problemas de optimización se pueden clasificar de la siguiente manera:

- Examination Timetabling Problem: el problema consiste en calendarizar un conjunto de evaluaciones (exámenes) en determinados horarios, salas de clases y supervisores (profesores) de tal forma que se satisfagan un conjunto de restricciones que varían de institución en institución. Usualmente, la programación de estas evaluaciones se realiza después que ha iniciado el periodo académico. Entre las restricciones que se suelen considerar se encuentran que un estudiante/profesor no tenga que rendir/supervisar dos pruebas el mismo día a la misma hora, que los salones de clases no se asignen a dos pruebas en el mismo día y hora, etc. Entre los indicadores de calidad de la planificación se consideran que el tiempo que transcurre entre dos pruebas programadas para el mismo día sea el mayor posible, y que los estudiantes, en la medida de lo posible, no tengan que rendir dos pruebas el mismo día.

- Course Timetabling Problem: el problema consiste en asignar los distintos cursos a distintos grupos de estudiantes, horas de clase y aulas, respetando restricciones tales como que ningún grupo de estudiantes debe tener dos clases en el mismo horario, que las aulas no tengan doble asignación, que se respete la capacidad de las aulas, entre otras. Asimismo, entre los indicadores de calidad se encuentran minimizar los desplazamientos de los estudiantes entre un aula y otra, se minimicen los espacios libres entre clases, que las materias catalogadas como "difíciles" se dicten en jornadas donde la retención cognitiva es mayor, etc. Por lo general, este problema se resuelve después de la matriculación de los estudiantes en los diversos grupos disponibles. 
- Staff Timetabling Problem: el problema consiste en asignar los profesores a las cátedras conforme a los horarios en los cuales se han planificado estas, respetando restricciones como que ningún profesor puede tener asignado dos clases a la misma hora el mismo día, que la carga de trabajo no puede exceder las normativas legales, y que la clase asignada debe respetar la disponibilidad horaria de los docentes, en especial, aquellos de tiempo parcial. Como indicadores de calidad se considera que los profesores deben ser asignados a las cátedras que mejor dominan, en los horarios de su preferencia y que no se asignen varias cátedras nocturnas todos los días a un mismo profesor.

Dada la metodología manual con la cual se elaboró la planificación académica en la institución de este estudio, el problema abordado sería una combinación de "coursetimetabling" con "staff-timetabling" debido a que ya se tienen asignados los horarios de clases, los profesores, y los estudiantes en las diversas cátedras, pero no las aulas. Por esta razón, el modelo propuesto se enfoca en resolver esta última etapa de la planificación, la asignación de las aulas, lo que podría definirse como "Room-Timetabling Problem".

\section{Estado del arte}

Los problemas de calendarización orientados a instituciones educativas han sido estudiados desde varios años atrás, por ejemplo (Gotlieb, 1963), por lo cual es un área de investigación multidisciplinaria bien establecida y madura, donde convergen disciplinas como la Investigación de Operaciones, Machine Learning y Ciencias de la Computación. Dada la importancia que tienen estos problemas en las operaciones de las instituciones educativas, varios trabajos se han publicado recientemente en la literatura científica (Carter \& Laporte, 1997; Asratian \& de Werra, 2002; Daskalaki, Birbas, \& Housos, 2004; FeiziDerakhshi, Babaei, \& Heidarzadeh, 2012; Babaei, Karimpour, \& Hadidi, 2015; Domenech \& Lusa, 2016; Akkan \& Gülcü, 2018; Yasari, Ranjbar, Jamili, \& Shaelaie, 2019), por mencionar algunos. A continuación, se explicará brevemente algunos de los trabajos más recientes y cuyas contribuciones destacan de cierta manera sobre aquellos publicados en la literatura.

Al-Yakoob \& Sherali (2007) formulan un modelo de calendarización con enfoque principal en la asignación de las aulas, cuya principal novedad es la consideración del flujo de 
estudiantes en los pasillos de los edificios de la institución educativa, el cual podría ser muy caótico, causando cogestión en estos y en los ascensores. Por esta razón, su modelo busca crear la planificación académica de tal manera que se minimice el flujo de estudiantes entre las aulas al cambiar de asignatura. Posteriormente, Domenech \& Lusa (2016) proponen un modelo matemático para planificar las cátedras de una institución superior donde la función objetivo de este busca minimizar la violación de las preferencias de los profesores, de tal forma que se este permita invertir más tiempo en la ejecución de otras actividades importantes, tales como los proyectos de investigación que realizan en la universidad.

Luego, Jamili, Hamid, Gharoun, \& Khoshnoudi (2018), a diferencia de otros modelos de calendarización que usualmente consideran una única función objetivo, proponen un modelo multiobjetivo en el cual se busca crear una calendarización que considere el cumplimiento de dos criterios que usualmente están contrapuestos: las preferencias de los profesores sobre los bloques horarios en los cuales desean dictar las clases y el tiempo disponible para la ejecución de proyectos de investigación. Dado que el modelo se formula de manera multiobjetivo, no se obtiene una única solución para el problema, sino un conjunto de soluciones no dominadas donde no es posible mejorar la calidad de un criterio sin sacrificar la calidad de otro. Con este conjunto de soluciones, el tomador de decisiones determinará cuál se ajusta mejor a las características propias de su institución.

En otro sentido, Akkan \& Gülcü (2018) proponen un modelo integrado de calendarización donde se consideran las decisiones de asignación de profesores a las cátedras y de estas a los diferentes bloques horarios. La principal novedad con respecto a otros modelos es la inclusión de la incertidumbre, la cual se representa como el hecho que un profesor, por razones de fuerza mayor, no pueda dictar la cátedra planificada en el horario establecido y solicite que se recalendarice la misma. Naturalmente, ante la presencia de este tipo de eventos, es deseable que la planificación realizada sea robusta, es decir, que se pueda afrontar el suceso realizando la menor cantidad de modificaciones posibles al esquema ya implementando.

Dado que los problemas de calendarización dependen mucho del contexto en el cual se desenvuelve la institución educativa, es complicado la generación de un modelo matemático general que abarque todas las particularidades de cada institución, más allá de 
las restricciones comunes tales como cruce de horarios, capacidad de aulas, entre otras. El lector interesado en conocer el abanico de modelos propuestos en la literatura, puede referirse a (Feizi-Derakhshi, Babaei, \& Heidarzadeh, 2012; Hosny, 2018; Vrielink, Jansen, Hans, \& van Hillegersberg, 2019).

\section{Modelo de asignación de aulas}

En esta sección se presenta el contexto específico del problema de asignación a resolver para posteriormente formular un modelo de programación lineal entera que encuentre una solución para el mismo de manera automática.

\section{ANÁLISIS DE LOS RESULTADOS}

\section{Descripción de los datos disponibles}

La Escuela de Computación y Telecomunicaciones de la Facultad de Ingeniería actualmente cuenta con 5 aulas para dictar clases, cuyas principales características se muestran en la Tabla 1. Actualmente, la Escuela oferta dos carreras de grado además de planificar las materias de ciencias físicas y matemáticas.

Tabla 1: Características de las aulas.

\begin{tabular}{ccc}
\hline Aula & Capacidad & Observaciones \\
\hline G201 & 35 & Pizarra mediana \\
G202 & 33 & $\begin{array}{c}\text { Pizarra grande } \\
\text { G203 }\end{array}$ \\
Pab. Cisco & 32 & $\begin{array}{c}\text { Aula alargada pequeña } \\
\text { e ideal para uso de laptops. } \\
\text { Aula pequeña ideal para clases } \\
\text { de electrónica o afines. }\end{array}$ \\
$\begin{array}{c}\text { Lab. de } \\
\text { Electrónica }\end{array}$ & 10 &
\end{tabular}

Fuente: Elaboración propia.

Para el presente periodo académico, la Escuela oferta 43 paralelos de diferentes asignaturas planificados acorde a la estimación de demanda realizada por el equipo de gestión académica. Una muestra de esta planificación se presenta en la Tabla 2. 
Tabla 2: Muestra resumida de la planificación académica.

\begin{tabular}{cccc}
\hline Código & Cátedra & $\begin{array}{c}\text { No. de } \\
\text { Paralelos }\end{array}$ & $\begin{array}{c}\text { Total de } \\
\text { Alumnos } \\
\text { Matriculados }\end{array}$ \\
\hline UCOM1357 & Robótica & 1 & 11 \\
UMAT102 & Matemáticas I & 4 & 70 \\
UFIS150 & Física II & 2 & 40 \\
UTEL433 & Teoría & 1 & 5 \\
\hline
\end{tabular}

Fuente: Elaboración propia.

En los problemas de calendarización, las restricciones generalmente tienden a separarse en dos grupos: las restricciones duras y las restricciones blandas. Las restricciones duras tienen una prioridad alta, y serán obligatorias en su satisfacción. De hecho, las calendarizaciones generalmente solo se considerarán factibles si y solo si se han satisfecho todas las restricciones duras del problema. Mientras tanto, las restricciones suaves son aquellas a las que se quiere obedecer si es posible. (Lewis \& Paechter, Finding feasible timetables using group-based operators, 2007) En este sentido, el objetivo por lograr consiste en, tomando como base la planificación académica ya realizada para el periodo académico por iniciar, asignar las aulas a las distintas duplas cátedra - paralelo de tal forma que se cumplan las siguientes restricciones fuertes:

- La capacidad de las aulas de clase no puede ser excedida, por tanto, no se pueden asignar parejas cátedra - paralelo cuya cantidad de alumnos matriculados excede la capacidad del aula.

- Para cada aula de clase y por cada bloque horario (combinación día - hora), el aula no puede tener asignada dos o más parejas cátedra - paralelo, puesto que esto provocaría un cruce de horas impidiendo la ejecución normal de las actividades de docencia.

- Cada pareja cátedra - paralelo debe tener asignada un aula de clase en el bloque horario establecido en la planificación académica, es decir, no se puede mover el horario de clase fijado por el equipo de gestión académica. 
Adicionalmente, se desean satisfacer en lo posible, las siguientes restricciones blandas:

- Se desea minimizar el uso de aulas que no pertenecen a la Escuela de Computación y Telecomunicaciones, pues esto implicaría que tanto los estudiantes como los profesores tengan que recorrer mayores distancias al cambiarse de una clase a otra.

- Se desea asignar a cada cátedra un aula que, de cierta manera, favorezca la presentación de los temas abordados en la misma. Por ejemplo, las cátedras relacionadas con electrónica deberían ser dictadas, de preferencia, en el Laboratorio de Electrónica. Las cátedras que requieren el uso constante de los proyectores y de los computadores personales de los estudiantes deberían asignarse al Laboratorio Cisco.

- Se desea que el Laboratorio Cisco tenga un mayor nivel de utilización en la jornada nocturna comparado con las aulas tradicionales de clase.

\section{Formulación del modelo matemático}

En este problema, como se discutió anteriormente, las restricciones duras deben cumplirse en cualquier circunstancia. Significa que, si se violan las restricciones duras, entonces el calendario es inviable y se descartará. Por otro lado, se pueden violar las restricciones blandas, sin embargo, tratamos de minimizar dicha violación para mejorar la calidad de la solución (Obit, 2010). Por lo que, con base en la problemática descrita en la Sección 1 y en la Sección 2, se procede a formular el modelo matemático que asignará las aulas a las distintas parejas cátedra - paralelo. Los conjuntos (objetos/entidades) que forman parte del modelo son los siguientes:

- $A$ es el conjunto de aulas disponibles.

- $M$ es el conjunto de materias o cátedras ofertados en el periodo académico.

- $\quad P$ es el conjunto de paralelos ofertados por cada materia.

- $H$ es el conjunto con los bloques de horas disponibles para dictar las asignaturas.

- $D$ es el conjunto de días de la semana. 
Los parámetros del modelo (datos conocidos con anticipación) son los siguientes:

- AlumMat $_{i, j}$ es la cantidad de alumnos matriculados en la cátedra $i \in M$, paralelo $j \in$ $P$.

- $\quad \operatorname{Asig}_{i, j}^{h, d}$ toma el valor de 1 si la cátedra $i \in M$, paralelo $j \in P$ está asignada al bloque $h \in H$ en el día $d \in D$.

- CapAula $_{a}$ indica la cantidad de alumnos que puede albergar el aula $a \in A$.

- $\quad A$ fin $_{i, a}$ es la idoneidad (estimada subjetivamente) que tiene el aula $a \in A$ para el dictado de la cátedra $i \in M$.

- CostAula $a$ es el costo/penalización (estimado subjetivamente) que se debe pagar por utilizar el aula $a \in A$, el cual es más alto cuando el aula no pertenece a la Escuela de Computación.

Las variables de decisión (elementos que se pueden controlar libremente) del modelo de optimización son:

- $\quad X_{a, i, j}^{d, h}=\left\{\begin{array}{l}1, \quad \text { si el aula a se asigna a la materia } i, \text { paralejo } j \text { en el día } d, \text { bloque } h \\ 0, \quad \text { caso contrario }\end{array}\right.$

- $\quad Z$ = valor total de la función objetivo

El modelo de optimización estaría dado por:

$\max z=\sum_{a \in A} \sum_{i \in M} \sum_{j \in P} \sum_{d \in D} \sum_{h \in H}\left(\right.$ Afin $\left._{i, a}-\operatorname{CostAula} a\right) X_{a, i, j}^{d, h}$

s.t:

$$
\begin{array}{ll}
\text { AlumMat }_{i, j} \cdot X_{a, i, j}^{d, h} \leq \text { CapAula }_{a} & \forall a \in A, i \in M, j \in P, d \in D, h \in H \\
\sum_{i \in M} \sum_{j \in P} X_{a, i, j}^{d, h} \leq 1 & \forall a \in A, d \in D, h \in H \\
\sum_{a \in A} X_{a, i, j}^{d, h}=1 & \forall i \in M, j \in P, d \in D, h \in H \mid A s i g_{i, j}^{d, h} \\
X_{a, i, j}^{d, h} \leq \text { Asig }_{i, j}^{d, h} & \forall a \in A, i \in M, j \in P, d \in D, h \in H \\
X_{a, i, j}^{d, h} \in\{0,1\} & \forall a \in A, i \in M, j \in P, d \in D, h \in H
\end{array}
$$


La ecuación (1) es la función objetivo del problema en la cual se busca maximizar la afinidad del aula para el dictado de las cátedras menos las penalizaciones por usar aulas que no son parte de la Escuela de Computación. Según la forma como se fijen los valores de los parámetros de la función objetivo, se incentiva al modelo a encontrar una solución que satisfaga las restricciones blandas del problema. La restricción dada por la ecuación (2) garantiza que el aula asignada tenga la capacidad suficiente conforme a la cantidad de alumnos matriculados en cada pareja asignatura - paralelo, por otro lado, la ecuación (3) asegura que no se asigne un aula a dos parejas cátedra - paralelo, evitando así los cruces horarios en las asignaciones de aulas. Asimismo, la ecuación (4) garantiza que cada pareja cátedra - paralelo debe tener asignada exactamente un aula en el bloque y día en el cual ha sido programada. Finalmente, la ecuación (5) establece que no se asignen aulas en los bloques y días donde ninguna materia ha sido programada mientras que la ecuación (6) presenta la naturaleza lógica de las variables de decisión.

La principal novedad del modelo matemático propuesto radica en la manera con la cual se fijan los parámetros de la función objetivo, en especial $A f_{i n_{i, a}}$, el cual permite establecer la idoneidad de las aulas para el dictado de las cátedras, considerando factores como la cantidad de proyectores, pizarras, capacidad, etc. De esta manera se incentiva al modelo matemático a asignar las aulas que mejor se adaptan a la naturaleza de las cátedras impartidas por la Facultad.

\section{Solución y resultados del modelo matemático}

Para la implementación y resolución del modelo de programación lineal entera definido por las ecuaciones (1) - (6) se utilizó el modelizador AIMMS Developer 4.68 en conjunto con el solver IBM-CPLEX versión 12.9 utilizando un computador personal Dell Inspiron 14R Intel Core i7 con 16GB de RAM. El tiempo de procesamiento computacional fue de 3.61 segundos logrando los resultados (que se muestran en parte) en la Tabla 3.

Tabla 3: Asignación de Aulas (Muestra)

\begin{tabular}{ccccc}
\hline Materia & Paralelo & Días & Hora & Aula \\
\hline UMAT192 & 3 & Lunes-Miércoles & $10 h 30-12 h 00$ & F208 \\
UMAT202 & 2 & Martes-Jueves & $10 h 30-12 h 00$ & F208 \\
\hline
\end{tabular}




\begin{tabular}{ccccc}
\hline UCOM487 & 81 & Lunes-Miércoles & $19 \mathrm{~h} 35-21 \mathrm{~h} 05$ & G201 \\
UFIS105 & 2 & Lunes-Miércoles & $10 \mathrm{~h} 30-12 \mathrm{~h} 00$ & G201 \\
UCOM250 & 1 & Lunes-Miércoles & 07h10-08h40 & G202 \\
UCOM320 & 1 & Martes-Jueves & $07 \mathrm{~h} 00-08 \mathrm{~h} 40$ & G202 \\
UMAT102 & 3 & Lunes-Miércoles & $10 \mathrm{~h} 30-12 \mathrm{~h} 00$ & G203 \\
UMAT102 & 4 & Martes-Jueves & $10 \mathrm{~h} 30-12 \mathrm{~h} 00$ & G203 \\
UCOM190 & 1 & Lunes-Miércoles & $10 \mathrm{~h} 30-12 \mathrm{h00}$ & LabElec \\
UELE402 & 81 & Martes-Jueves & $18 \mathrm{~h} 00-19 \mathrm{~h} 30$ & LabElec \\
UCOM1357 & 81 & Lunes-Miércoles & $18 \mathrm{~h} 00-19 \mathrm{~h} 30$ & Netw \\
UCOM252 & 1 & Lunes-Miércoles & $08 \mathrm{~h} 45-10 \mathrm{~h} 15$ & Netw \\
\hline
\end{tabular}

Fuente: Elaboración propia.

La asignación de aulas obtenida con el modelo matemático produjo los siguientes indicadores de calidad: (1) el nivel de utilización del aula G-202, la más grande y equipada de la escuela subió del $30 \%$ al $75 \%$, de tal manera que la mayoría de las cátedras de ciencias físicas y matemáticas se dictan en dicha aula. Para evitar el desgaste de sus instalaciones, su uso se limitó durante la jornada nocturna. (2) El nivel de utilización del Laboratorio CISCO aumentó un 100\% durante la jornada matutina, dada su idoneidad para el dictado de cátedras que requieren el uso constante de computadoras. Anteriormente, este pasaba desocupado en dicha jornada, siendo principalmente utilizado en la jornada nocturna. (3) La satisfacción de los docentes con la asignación de las aulas estuvo en el $90 \%$, la cual es una mejora sustancial con respecto al indicador anterior (20\%), debido a que se está asignadas las aulas sin darle preferencia especial a ningún profesor, sino más bien a la naturaleza de la cátedra y la cantidad de estudiantes matriculados y, (4) el tiempo utilizado para la asignación de las aulas fue menor a 2 minutos, comparado con las horas de trabajo manual necesarias para la ejecución de la misma tarea.

\section{CONCLUSIONES Y RECOMENDACIONES}

En el presente trabajo de investigación se propuso un nuevo modelo de programación lineal entera para resolver el problema de asignación de aulas de clase a las cátedras impartidas por la Escuela de Computación y Telecomunicaciones de una institución de educación superior. La principal novedad de este modelo con respecto a otros presentados en la literatura radica en la función objetivo de este, en la cual se busca maximizar la afinidad 
total del emparejamiento aula - cátedra respetando las restricciones de capacidad y cruce de horas, lo cual produce una asignación de aulas más equitativa y en menor tiempo de procesamiento. Así mismo se logra aumentar el nivel de satisfacción de los docentes y el nivel de utilización de las aulas emblemáticas, cuidando al mismo tiempo que estas no se deterioren por uso excesivo.

Como trabajo futuro se pueden considerar dos posibilidades, la primera consiste en la formulación de un modelo matemático integrado que resuelva al mismo tiempo la calendarización de las clases, la asignación de profesores y la asignación de las aulas, previo al registro de los estudiantes, mientras que la segunda radica en incluir la incertidumbre dentro del modelo, de tal forma que permita responder de manera efectiva a eventos inesperados tales como que un aula se inhabilite, entre otros.

\section{REFERENCIAS BIBLIOGRÁFICAS}

Akkan, C., \& Gülcü, A. (2018). A bi-criteria hybrid Genetic Algorithm with robustness objective for the course timetabling problem. Computers \& Operations Research, 22 - 32.

Al-Betar, M., Khader, A., \& Zaman, M. (2012). University course timetabling using a hybrid harmony search metaheuristic algorithm. IEEE Transactions on Systems, Man, and Cybernetics, Part C (Applications and Reviews), 664 - 681.

Al-Yakoob, S. M., \& Sherali, H. D. (2007). A mixed-integer programming approach to a class timetabling problem: A case study with gender policies and traffic considerations. European Journal of Operational Research, 1028 - 1044.

Asratian, A., \& de Werra, D. (2002). A generalized class-teacher model for some timetabling problems. European journal of operational research, 531 - 542.

Babaei, H., Karimpour, J., \& Hadidi, .. (2015). A survey of approaches for university course timetabling problem. Computers \& Industrial Engineering, 43 - 59.

Carter, M. W., \& Laporte, G. (1997). Recent Developments in Practical Course Timetabling. international conference on the practice and theory of automated timetabling, 3 - 19.

CES. (21 de 09 de 2019). Gaceta. Recuperado de gaceta.ces.gob.ec: http://gaceta.ces.gob.ec/inicio.html?id_documento=234533 
Dantzig, G., \& Thapa, M. (2006). Linear programming 1: introduction. Springer.

Daskalaki, S., Birbas, T., \& Housos, E. (2004). An integer programming formulation for a case study in university timetabling. European Journal of Operational Research, 117 - 135.

Domenech, B., \& Lusa, A. (2016). A MILP model for the teacher assignment problem considering teachers' preferences. European Journal of Operational Research, $1153-1160$.

El Universo. (16 de Abril de 2019a). Tiempos se acortan en carreras de tercer nivel en Ecuador. Recuperado de El Universo: https://bit.ly/2mb5HzP

El Universo. (14 de Julio de 2019b). Estudiantes y docentes se adaptan a la educación pública en línea en Ecuador. Recuperado de El Universo: https://bit.ly/2kAFc6L

El Universo. (2 de Septiembre de 2019c). 330 ítems comprende la quinta evaluación externa a 55 centros de estudios superiores del Ecuador. Recuperado de El Universo: https://bit.ly/2m9FDVN

Feizi-Derakhshi, M. R., Babaei, H., \& Heidarzadeh, J. (2012). A survey of approaches for university course timetabling problem. In Proceedings of 8th international symposium on intelligent and manufacturing systems (págs. 307 - 321). Adrasan: Sakarya University Department of Industrial Engineering.

Goh, S. L., Kendall, G., \& Sabar, N. R. (2019). Simulated annealing with improved reheating and learning for the post enrolment course timetabling problem. Journal of the Operational Research Society, 873 - 888.

Gotlieb, C. C. (1963). The construction of class-teacher timetables. IFIP congress (págs. 73 - 77). IFIP.

Hernández, R., Miranda, J., y Rey, P. (2008). Programación de Horarios de Clases y Asignación de Salas para la Facultad de Ingeniería de Universidad Diego Portales mediante un Enfoque de Programación Entera. Revista Ingeniería de Sistemas, 121 - 141.

Hillier, F., Lieberman, G., y Osuna, M. (2010). Introducción a la Investigación de Operaciones. McGraw-Hill.

Hosny, M. (2018). Metaheuristic Approaches for Solving University Timetabling Problems: A Review and Case Studies from Middle Eastern Universities. International Conference Europe Middle East \& North Africa Information Systems and Technologies to Support Learning , $10-20$. 
Jamili, A., Hamid, M., Gharoun, H., \& Khoshnoudi, R. (2018). Developing a Comprehensive and Multi-Objectivemathematical model for university course timetabling problem: a real case study. Conference: proceedings of the international conference on industrial engineering and operations management (pág. 2108). Paris: Industrial Engineering and Operations Managemen.

June, T. L., Obit, J. H., Leau, Y. B., \& Bolongkikit, J. (2019). Implementation of Constraint Programming and Simulated Annealing for Examination Timetabling Problem. Computational Science and Technology, 175 - 184.

Lewis, R. (2008). A survey of metaheuristic-based techniques for university timetabling problems. OR spectrum, $167-190$.

Lewis, R., \& Paechter, B. (2007). Finding feasible timetables using group-based operators. IEEE Transactions on Evolutionary Computation, 397 - 413.

Lindahl, M., Mason, A. J., Stidsen, T., \& Sørensen, M. (2018). A strategic view of University timetabling. European Journal of Operational Research, 35 - 45.

McCollum, B. (2006). A Perspective on Bridging the Gap Between Theory and Practice in University Timetabling. International Conference on the Practice and Theory of Automated Timetabling (págs. 3 - 23). Berlin: Springer.

MirHassani, S. (2006). A computational approach to enhancing course timetabling with integer programming. Applied Mathematics and Computation, 814 - 822.

Müller, T., Rudová, H., \& Barták, R. (2004). Minimal perturbation problem in course timetabling. International Conference on the Practice and Theory of Automated Timetabling (págs. 126 - 146). Heidelberg: Springer.

Nategh, M. N., Hosseinabadi, A. R., \& Balas, V. E. (2018). University-timetabling problem and its solution using GELS algorithm: a case study. International Journal of Advanced Intelligence Paradigms, 368 - 377.

Obit, J. H. (2010). Developing novel meta-heuristic, hyper-heuristic and cooperative search for course timetabling problems. Nottingham: University of Nottingham.

Obit, J. H., Ouelhadj, D., Landa-Silva, D., Vun, T. K., \& Alfred, R. (2011). Designing a multiagent approach system for distributed course timetabling. 11th International Conference on Hybrid Intelligent Systems (HIS) IEEE (págs. 103 - 108). IEEE.

Phillips, A. E., Walker, C. G., Ehrgott, M., \& Ryan, D. M. (2017). Integer programming for minimal perturbation problems in university course timetabling. Annals of Operations Research, 283 - 304. 
Pinedo, M. (2016). Scheduling. Springer.

Sandhu, K. (2003). Automating class schedule generation in the context of a university timetabling information system. Brisbane: Griffith University.

Sarin, S. C., Wang, Y., \& Varadarajan, A. (2010). A university-timetabling problem and its solution using Benders' partitioning-a case study. Journal of Scheduling, $131-141$.

Schaerf, A. (1999). A survey of automated timetabling. Artificial intelligence review, 87 - 127.

Song, L., Liu, S., Tang, X., Peng, X., \& Chen, M. (2018). An iterated local search algorithm for the University Course. Applied Soft Computing, 597 - 608.

Vrielink, R. O., Jansen, E. A., Hans, E. W., \& van Hillegersberg, J. (2019). Practices in timetabling in higher education institutions: a systematic review. Annals of operations research, 145 - 160.

Weitz, R. R., \& Jelassi, M. T. (1992). Assigning students to groups: a multi-criteria decision support system approach. Decision Sciences, 746 - 757.

Wolsey, L. (1998). Integer Programming. Wiley.

Yasari, P., Ranjbar, M., Jamili, N., \& Shaelaie, M. H. (2019). A two-stage stochastic programming approach for a multi-objective course timetabling problem with courses cancelation risk. Computers \& Industrial Engineering, 650 - 660. 